
Not for reproduction, distribution or commercial use.

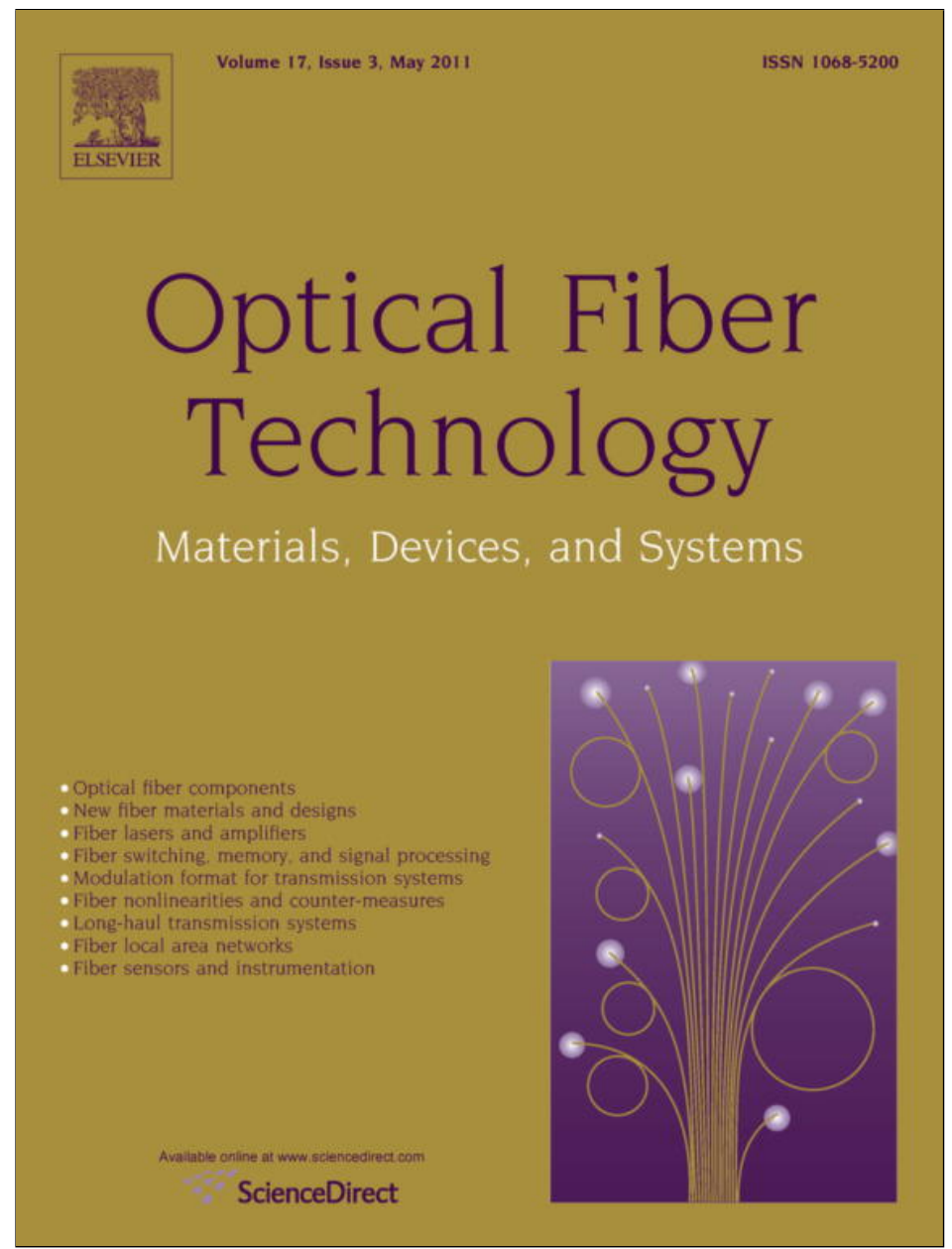

This article appeared in a journal published by Elsevier. The attached copy is furnished to the author for internal non-commercial research and education use, including for instruction at the authors institution and sharing with colleagues.

Other uses, including reproduction and distribution, or selling or licensing copies, or posting to personal, institutional or third party websites are prohibited.

In most cases authors are permitted to post their version of the article (e.g. in Word or Tex form) to their personal website or institutional repository. Authors requiring further information regarding Elsevier's archiving and manuscript policies are encouraged to visit:

http://www.elsevier.com/copyright 


\title{
Modal four-wave mixing supported generation of supercontinuum light from the infrared to the visible region in a birefringent multi-core microstructured optical fiber
}

\author{
Gabriele Manili a,*, Daniele Modotto ${ }^{a}$, Umberto Minoni ${ }^{a}$, Stefan Wabnitz ${ }^{a}$, Costantino De Angelis ${ }^{a}$, \\ Graham Town $^{\mathrm{b}}$, Alessandro Tonello ${ }^{\mathrm{c}}$, Vincent Couderc ${ }^{\mathrm{c}}$ \\ a Dipartimento di Ingegneria dell'Informazione, Università degli Studi di Brescia, via Branze 38, 25123 Brescia, Italy \\ ${ }^{\mathrm{b}}$ Department of Physics and Engineering, Faculty of Science, Macquarie University, NSW 2109, Australia \\ ' XLIM UMR CNRS 6172, Université de Limoges, 87060 Limoges, France
}

\section{A R T I C L E I N F O}

\section{Article history:}

Received 29 October 2010

Revised 21 January 2011

Available online 22 February 2011

\section{Keywords:}

Nonlinear optics

Microstructured optical fiber

Supercontinuum

Four-wave mixing

\begin{abstract}
A B S T R A C T
We experimentally studied the process of supercontinuum generation in a birefringent multi-core microstructured optical fiber. By selecting the excitation of the fundamental mode, or by combining the first and the second order modes of a particular core, it was possible to emphasize the role of four-wave mixing on the transfer of power from the infrared to the visible region of the spectrum. We carried out an in-depth analysis of the effects of input light polarization on the generated supercontinuum spectral features. The measured polarization properties of the output Stokes and anti-Stokes bands confirmed the strong vector nature of the four-wave mixing processes. The experimental spectra exhibit excellent agreement with numerical simulations of the nonlinear mode interactions.
\end{abstract}

(c) 2011 Elsevier Inc. All rights reserved.

\section{Introduction}

Supercontinuum (SC) generation is a commonplace, but yet fascinating and in some aspects still unexplained phenomenon that is observed whenever high-power light waves are injected in a nonlinear medium (e.g. an optical fiber). Optical radiation gets spectrally broadened in a dramatic fashion as a result of the a complex interplay between linear and nonlinear responses [1]. In recent years microstructured optical fibers (MOFs) have been extensively used for the purpose of SC generation, as they enjoy high field confinement and large flexibility in the tailoring of the dispersion profile, so that the zero dispersion wavelength (ZDW) may be even shifted down into the visible range. In optical fibers $\mathrm{SC}$ is typically initiated by modulation instability (MI) of the quasi-continuous pump wave. The subsequent spectral broadening in the near infrared (NIR) is then ascribed to optical soliton formation and the associated Raman self-frequency shift. Whereas SC development in the normal dispersion regime and in the visible spectrum is associated with cross-phase modulation (XPM), fourwave mixing (FWM) with the corresponding NIR spectral components of the SC [2-8], or dispersive wave generation [9].

In spite of the extensive use of MOFs for research purposes, their applications to practical SC sources is until now limited by

\footnotetext{
* Corresponding author.

E-mail address: gabriele.manili@ing.unibs.it (G. Manili).
}

the maximum spectral brightness that can be obtained under single-core excitation (before the damage threshold of the fiber input end is reached). This observation motivates the interest in novel, high spectral brightness SC sources such as those based on multi-core MOFs. In principle, such fibers could lead to coherent/ incoherent recombination of the SC that is individually generated by each core. As a first step in this direction, in this work we studied SC generation in a seven-core MOF. Note that the different geometries of the cores may lead to a substantial extension of the overall SC spectral range with respect to single-core MOFs. To this end, we characterized the spectral features of the SC that was obtained by individually exciting the different MOF cores. For symmetry reasons, it was sufficient to consider only three out of the seven cores. For this purpose we used a compact, low cost commercial nanosecond Nd:YAG Q-switched laser pump at $1064 \mathrm{~nm}$. We performed a complete analysis of the SC characteristics as a function of the input pump polarization and power, as well as of the modal excitation conditions. Extensive comparisons between experiments and simulations provided a systematic analysis of the polarization properties of the generated SC light.

As a matter of fact, it was previously pointed out in Ref. [2] that modal FWM may lead to a bright visible SC. SC generation based on nonlinear interactions among different coupled fiber modes was also numerically analyzed in Ref. [10], where the propagation of femtosecond pulses in a holey optical fiber was simulated. To our knowledge, in our work we obtain the first experimental evidence 
of SC generation in MOFs under the simultaneous and efficient excitation on the two axes of birefringence of both the fundamental $L P_{01}$ and the higher-order $L P_{11}$ fiber modes (hereafter called intermodal vector excitation). As we shall see, under such pumping conditions the SC band-width could be substantially extended into the visible range of the spectrum. In addition, by using the input pump polarization degree of freedom we could obtain a nearly symmetric redistribution of the SC energy among the NIR and visible regions. In fact, vector FWM leads to three pairs of coupled sidebands in the NIR and in the visible that may provide a flexible shaping of the SC spectral broadening in each of their respective spectral regions.

This paper is organized as follows: In Section 2 we highlight the characteristics of the nonlinear multi-core MOF that was used in the experiments. In Section 3 we describe the experimental setup and the different SC generation results obtained when exciting the three representative cores of the seven-core MOF. A more detailed discussion is devoted to the case of central core excitation. Indeed, as we shall see, this configuration leads to the experimental evidence of intermodal vector FWM-activated SC. Our results are summarized in Section 4.

\section{Characteristics of the microstructured optical fiber}

Our multi-core silica fiber was manufactured by PERFOS, in collaboration with the XLIM Research Institute of Limoges, by employing the stack and draw technique. By using the Scanning Electron Microscope (SEM) image of the multi-core MOF (see Fig. 1) and a commercial electromagnetic field solver (COMSOL Multiphysics) we calculated the dispersion curves of the guided modes and could infer that the seven cores of the fiber were effectively decoupled. Each of the cores acted like an independent highly birefringent fiber.

We limit our analysis to the highlighted cores in Fig. 1: for symmetry reasons, their excitation leads to all types of SC that can be generated when using this fiber. We name these cores as "lateral", "central" and "off-axis" when moving from left to right in Fig. 1, respectively. The asymmetry created by the two central holes leads to highly birefringent lateral and central cores: their principal axes are parallel to the $x$ and $y$ directions. In fact, higher core ellipticity leads to higher values of phase and group birefringence [11]. The

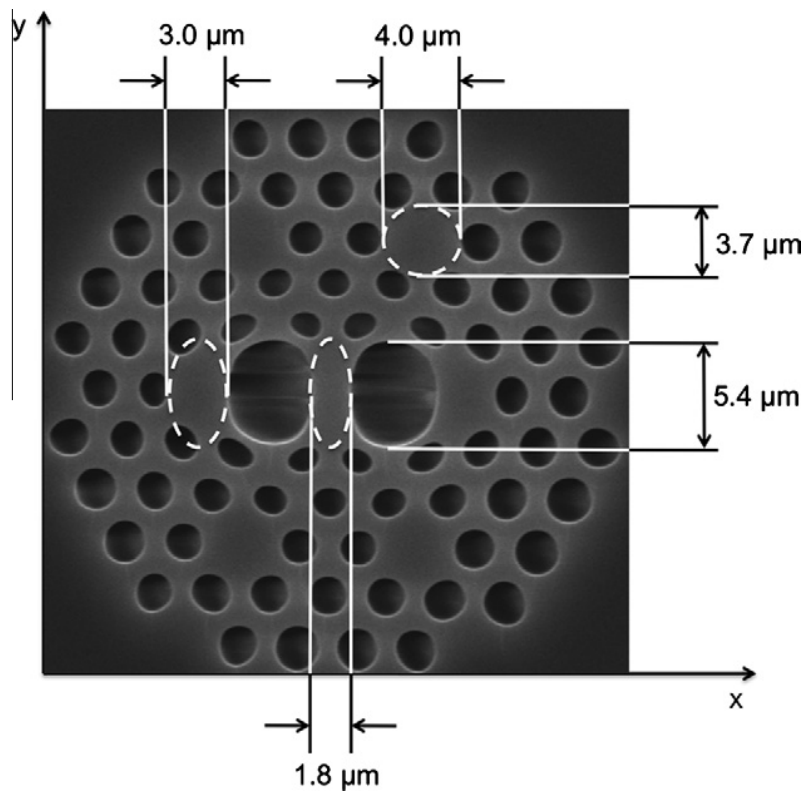

Fig. 1. SEM image of the MOF cross-section. SC generation was studied under excitation of the circled cores. phase birefringence $B_{\Phi}$ is defined as the difference between the effective refractive indices of the two fundamental modes polarized along the $x$ and $y$ axes. Whereas the group birefringence $B_{g}$ is the difference of their group indices. At the excitation wavelength of $1064 \mathrm{~nm}$ we obtain $B_{\Phi}=-1.7 \times 10^{-3}$ and $B_{g}=2.7 \times 10^{-3}$ for the central core and $B_{\Phi}=-0.43 \times 10^{-3}$ and $B_{g}=0.73 \times 10^{-3}$ for the lateral core, respectively. The off-axis core exhibits the relatively low phase and group birefringence $B_{\Phi}=0.03 \times 10^{-3}$ and $B_{g}=-0.05 \times 10^{-3}$.

Four distinct modes are guided in the cores at the pump wavelength of $1064 \mathrm{~nm}$, namely a fundamental $L P_{01}$ and a second order $L P_{11}$ mode oriented along each of the two principal birefringence axes. The dispersion curves of central core modes $L P_{01}$ and $L P_{11}$ with different polarizations are shown in Fig. 2. As it can be seen, for each and every core of the fiber propagation occurs in the anomalous dispersion region at the pump wavelength. By adjusting the vertical and horizontal position of the focused pump beam at the input face of the multi-core fiber it was possible to excite each individual mode in the different cores, as well as a mixture of different modes [12].

\section{Experimental results}

In this section we describe SC generation experiments using the multi-core MOF. We considered first the excitation of the off-axis core: in this case the pump spectral broadening is mainly due to pump MI-seeded self-Raman scattering. Next we considered the excitation of the lateral core: in this case a weak FWM process could be observed under certain launch conditions. Yet, no light in the visible range of the spectrum was generated when using this configuration. At last when exciting the central core we observed a broad continuum extending from visible to IR wavelengths originating from a complex interplay of efficient modal FWM, MI and Raman scattering.

In our experiments we used a Nd:YAG Q-switched laser pump delivering $600 \mathrm{ps}$ pulses with $6 \mathrm{kHz}$ repetition rate at $1064 \mathrm{~nm}$. The peak power of the pump pulses was about $7 \mathrm{~kW}$. Such type of pump was previously employed for SC generation using MOFs with a few micrometer core radius [2,13-16]. Different pump coupling efficiencies were obtained for each individual mode or mode combination. The coupled power into the fiber was measured by using a broadband power meter at its output. Fig. 3 provides a schematic of the experimental setup.

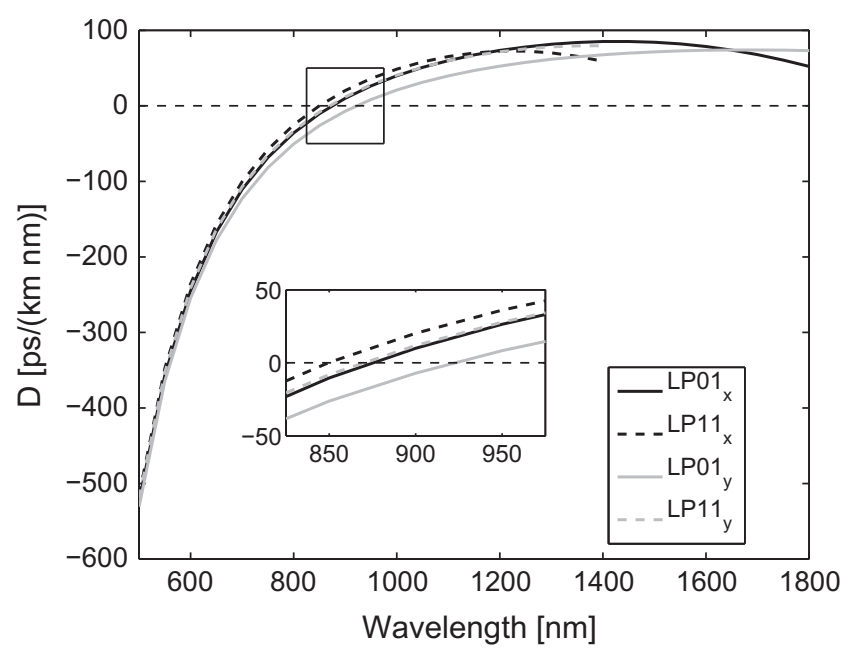

Fig. 2. Numerically calculated dispersion coefficients for the first two spatial modes of the central core of the MOF with a polarization state oriented along the two principal axes of the birefringence. The region around the mode ZDWs is magnified in the inset. 


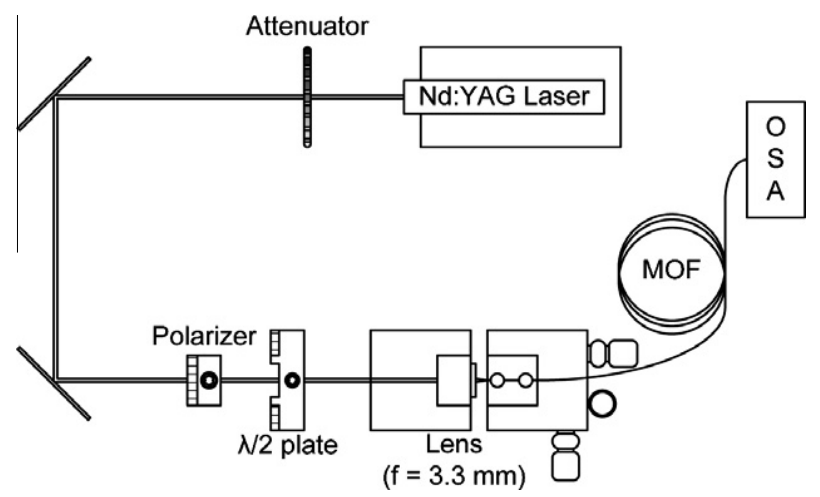

Fig. 3. Schematic of the experimental setup.

We used various optical components in order to control both power and polarization state of the input pump source which was lens-coupled into the MOF. The input lens focal length $(f=3.3 \mathrm{~mm})$ was chosen in order to obtain a MOF-compatible spot size and numerical aperture. The input beam was attenuated and passed through a half-wave plate in order to rotate the input state of polarization. The fiber was laid on a groove mounted on a 3-axis translation stage which could be moved with micrometric precision with respect to the lens. For spectral measurements we employed two different optical spectrum analyzers (ANDO AQ-6310B and ANRITSU MS9710C). The spectra presented in the following subsections were obtained with various fiber lengths, ranging from 1.8 to $2.5 \mathrm{~m}$, as specified in the text. Where not expressed differently, the spectra presented in this paper were acquired with the ANRITSU MS9710C operating with a resolution of $0.1 \mathrm{~nm}$ and a video band-width of $10 \mathrm{~Hz}$ (without averaging) in high dynamic range mode.

\subsection{Continuum generation in the off-axis core}

The spectrum that was obtained whenever the off-axis $L P_{01, x}$ mode was excited with maximum input power in a $1.8-\mathrm{m}$-long piece of fiber is shown in Fig. 4. For this core the coupled peak power was estimated at about $1 \mathrm{~kW}$. The ZDWs of the two propagating modes were very close to each other: $972 \mathrm{~nm}$ for the $L P_{01, x}$ and $971 \mathrm{~nm}$ for the $L P_{01, y}$. No significant difference in the SC spectrum could be detected when the polarization state of the input

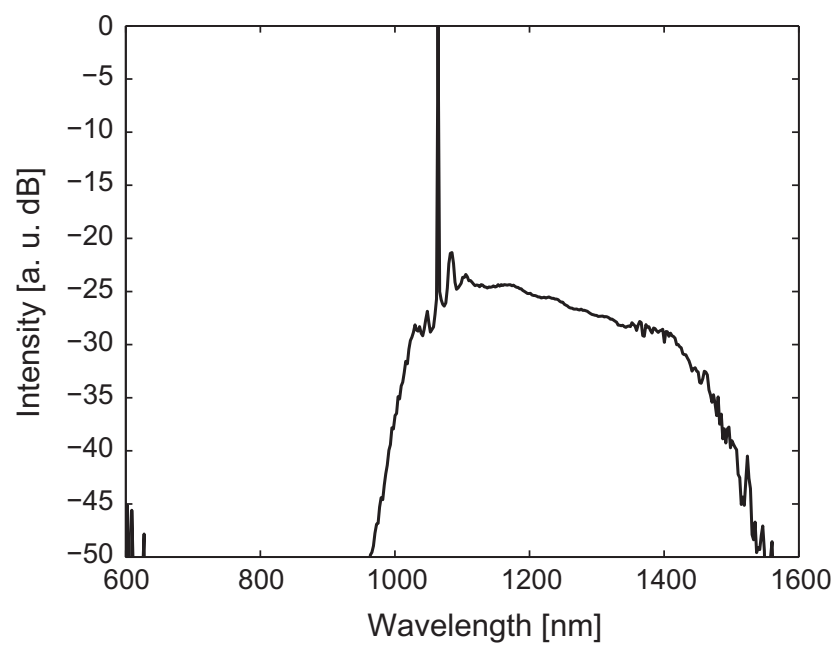

Fig. 4. (Fiber length $=1.8 \mathrm{~m}$ ) SC spectrum for the off-axis core as measured at full power. beam was rotated by $90^{\circ}$. Since the pump wavelength falls in the anomalous dispersion region of the mode, the SC is activated by pump MI (whose signature is provided by the small sidebands close to the residual pump in Fig. 4). Further spectral broadening towards the infrared is ascribed as usual to the generation of red-shifting solitons under the action of Raman self-scattering.

\subsection{Continuum generation in the lateral core}

Let us describe now SC generation when coupling the pump into the lateral core modes of the MOF. Fig. 5 shows the intensity distribution of the two modes that propagate in the lateral core at $1064 \mathrm{~nm}$ for the $x$ polarization, i.e. the $L P_{01, x}$ (a) and the $L P_{11, x}$ (b) modes, respectively. Virtually identical intensity distributions are obtained for $y$ polarization modes. The ZDWs of the modes were the following: $942 \mathrm{~nm}\left(L P_{01, x}\right), 959 \mathrm{~nm}\left(L P_{01, y}\right), 835 \mathrm{~nm}$ $\left(L P_{11, x}\right)$ and $839 \mathrm{~nm}\left(L P_{11, y}\right)$.

For all measurements presented in this section, the peak coupled power in the lateral core of the MOF was estimated to be around $2.3 \mathrm{~kW}$, and the fiber length was $1.8 \mathrm{~m}$. SC spectra obtained by focusing the laser beam at full power into the center of the lateral core when selectively exciting only the $L P_{01, k}$ mode ( $k$ stands for the $x$ or $y$ polarization) are shown in Fig. 6 .

Whenever each $L P_{01}$ mode was individually excited the resulting SC spectrum was very similar to what observed when exciting the off-axis core. Indeed, also in this case the SC generation mechanism can be described as due to MI-induced pump break-up into solitons. The resulting soliton Raman self-frequency shift leads to spectral broadening into the infrared part of the spectrum.

By slightly moving (with respect to the previous situation) the input spot across the vertical direction it was possible to excite a combination of both $L P_{01}$ and $L P_{11}$ modes. In the case of bimodal excitation it was possible to observe the growth of a weak FWMgenerated signal at the short wavelengths edge of the spectrum, as shown in Fig. 7. The spectral location of this signal depends upon the polarization of the pump. When the input polarization was aligned along the $x$ axis, the FWM signal peak was obtained at $728 \mathrm{~nm}$; a $90^{\circ}$ rotation of the input pump shifted the FWM peak to $705 \mathrm{~nm}$.

The observed positions of the FWM spectral peaks are in good agreement with the phase-matching condition that governs intermodal FWM [2]:

$k_{P, 01}+k_{P, 11}-k_{S, 01}(\Omega)-k_{A, 11}(\Omega)=0$,

where the wave vectors $k$ are identified by $P, S$ and $A$ indices for the pump, Stokes and anti-Stokes waves, and by the indices of the corresponding modes, $L P_{01}$ and $L P_{11}$, respectively. The variable $\Omega$ indicates the sideband frequency detuning from the pump. In Eq. (1) all waves have equal polarization, which is determined by the orientation of the pump linear polarization along either the $x$ or $y$ axis. Eq. (1) predicts $728(707) \mathrm{nm}$ for the wavelength of the anti-Stokes wave whenever the pump light is polarized along the $x(y)$ axis, respectively. The corresponding Stokes wave falls in the far infrared, namely beyond $2 \mu \mathrm{m}$ or outside the range of our measuring instruments. Note that the Stokes wave is generated in a region where material absorption is high, which explains the observed lack of efficiency of the FWM process when exciting the lateral core. On the other hand, in the next section we shall describe the case of an efficient intermodal FWM process which successfully contributes to the spectral broadening process.

\subsection{Continuum generation in the central core}

In order to obtain the broadest spectrum of light, which extends from the visible part of the spectrum into the infrared, it was necessary to excite the modes of the central core of the fiber. Indeed in 


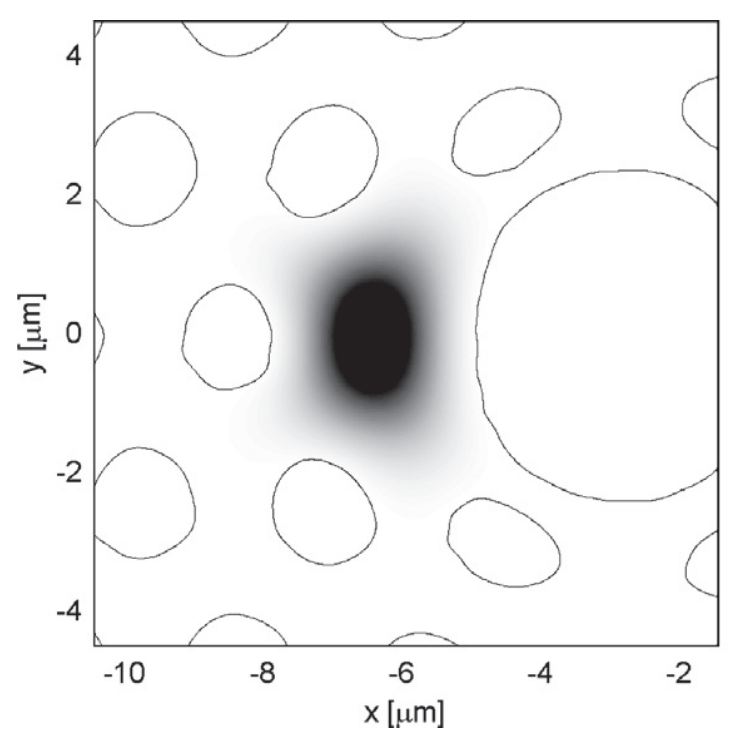

(a) $L P_{01, x}$

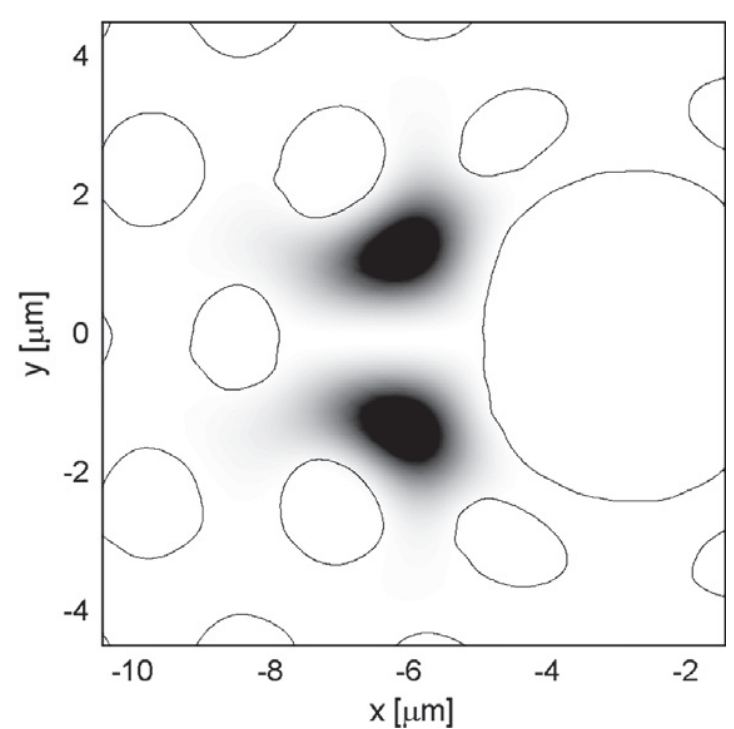

(b) $L P_{11, x}$

Fig. 5. Intensity distribution of the two $x$-polarized modes in the lateral core at $1064 \mathrm{~nm}$. Intensity is shown in a gray scale: highest field intensity corresponds to the darkest area. Thin lines represent the air-glass interfaces of the MOF internal structure.
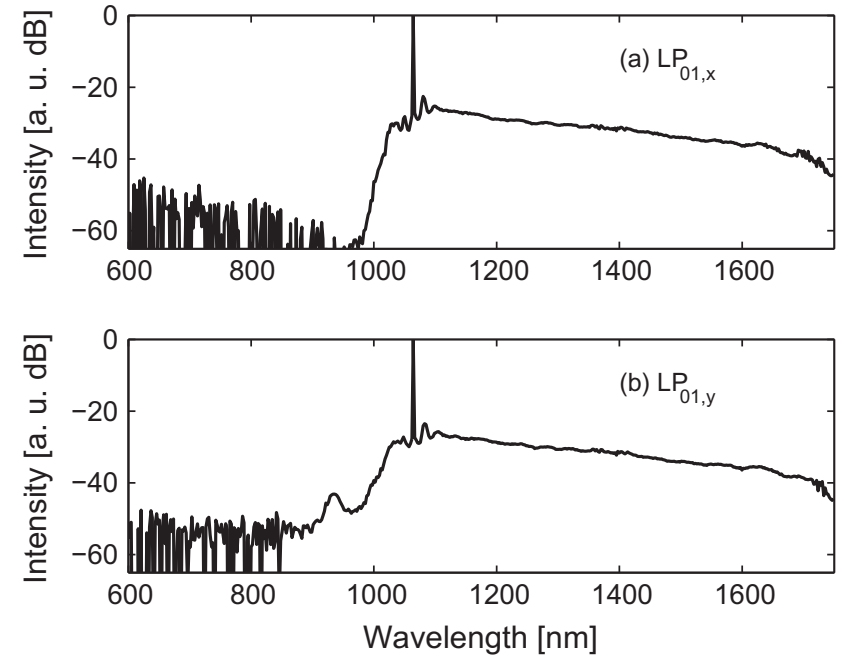

Fig. 6. (Fiber length $=1.8 \mathrm{~m}$ ) SC generation from the lateral core when only the (a) $L P_{01, x}$ or (b) $L P_{01, y}$ mode is excited.

this case, under certain launching conditions as discussed later, the efficiency of the modal FWM process was greatly enhanced, which in turn led to extreme spectral broadening. In Fig. 8 we show the shapes of the two central core modes for the $x$ polarization: the $L P_{01, x}$ (a) and $L P_{11, x}$ (b) at $1064 \mathrm{~nm}$. Similar shapes are obtained for the $y$ polarization. The ZDWs of the modes were the following: $876 \mathrm{~nm}\left(L P_{01, x}\right), 923 \mathrm{~nm}\left(L P_{01, y}\right), 849 \mathrm{~nm}\left(L P_{11, x}\right)$ and $870 \mathrm{~nm}\left(L P_{11, y}\right)$.

Fig. 9 shows the experimentally obtained spectrum under simultaneous excitation of the $L P_{01, y}$ and the $L P_{11, y}$ modes for a fiber length of $2.5 \mathrm{~m}$ (here the peak power coupled into the core was estimated to be around $1.6 \mathrm{~kW}$ ). In the same figure we also show the corresponding numerical simulation of the SC spectrum. The simulations are based on the numerical solution by the split-step Fourier method of the two coupled generalized nonlinear Schrödinger equations for each guided mode $[1,17]$. The numerically calculated dispersion curves were introduced in the numerical scheme by solving the dispersion operator in the frequency
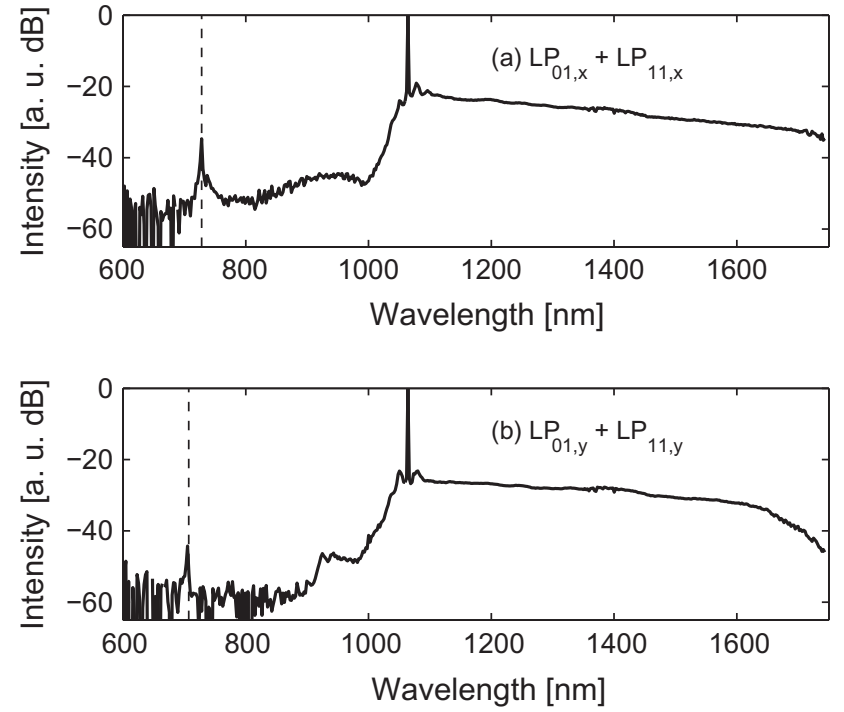

Fig. 7. (Fiber length $=1.8 \mathrm{~m}$ ) SC generation under lateral core excitation when a combination of the (a) $L P_{01, x}$ and $L P_{11, x}$ or (b) $L P_{01, y}$ and $L P_{11, y}$ modes was excited; markers indicate the positions of the FWM peaks as predicted by the phase matching condition.

domain. The nonlinear terms included self-phase modulation, cross-phase modulation and the scalar Raman response of silica glass [18]. We neglected the wavelength dependence of the modal effective areas and of the overlap coefficients. We assumed a constant value of $\gamma=21 \times 10^{-3} \mathrm{~W}^{-1} \mathrm{~m}^{-1}$. A small amount of gaussian noise was directly added to the input pulse in order to seed the nonlinear conversion process. It was not practical to simulate the propagation of 600 ps wide pulses because such temporal width posed severe constraints on the minimum number of temporal sample points that would be required in order to compute the pulse evolution and break-up in time domain, as well as spectral broadening in the frequency domain. As a consequence, we simulated the propagation of $60 \mathrm{ps}$ wide pulses and observed their spectrum at the fiber output for different levels of input power. 


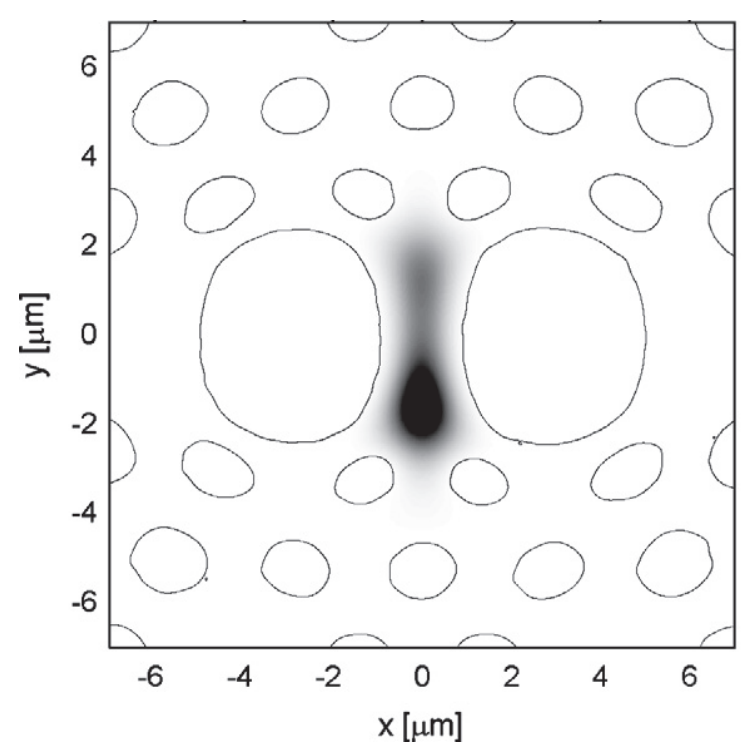

(a) $L P_{01, x}$

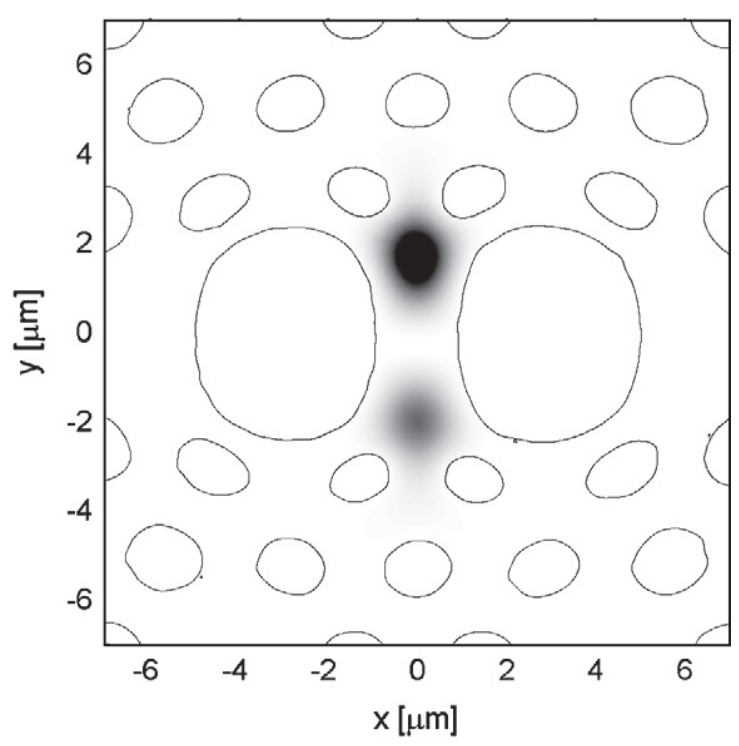

(b) $L P_{11, x}$

Fig. 8. Shapes of the modes in the central core at $1064 \mathrm{~nm}$. Intensity is shown in a gray scale: the highest field intensity corresponds to the darkest area. Thin lines represent the air-glass interfaces of the MOF internal structure.
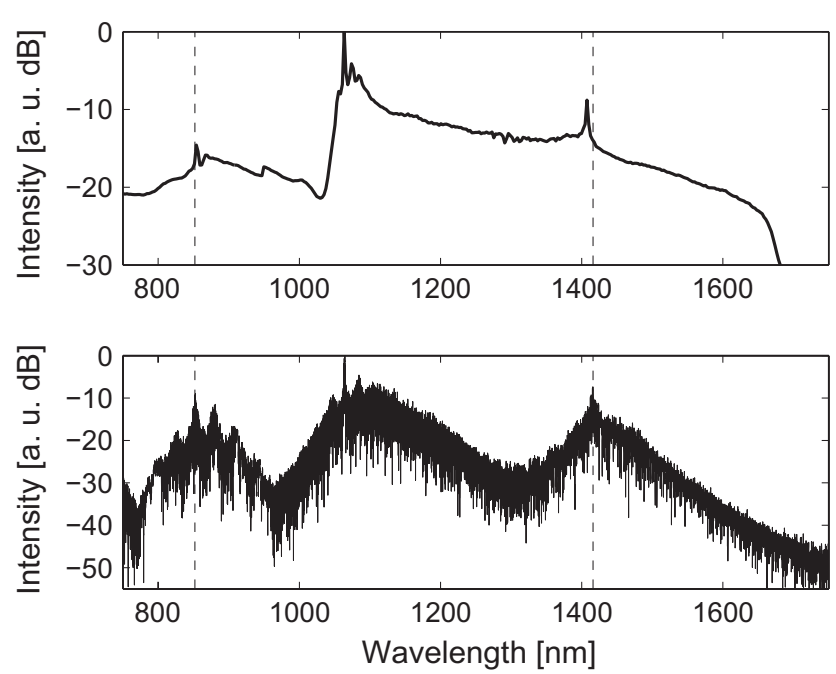

Fig. 9. Experimental (top, fiber length $=2.5 \mathrm{~m}$ ) and numerical (bottom, fiber length $=1.5 \mathrm{~m}, 800 \mathrm{~W}$ per mode) $\mathrm{SC}$ spectrum obtained with the simultaneous excitation of the central core $L P_{01, y}$ and $L P_{11, y}$ modes; markers indicate the ideal peak wavelengths from the phase-matching condition.

All simulations shown in this paper were obtained for a total pump input power of $1600 \mathrm{~W}$. Since the simulated pulses were shorter than in the experiment the temporal walk-off between the different spectral components due to group-velocity dispersion reduced the effective length for nonlinear frequency conversion. Yet we could obtain a remarkable qualitative agreement with the experimental results by simply using a relatively shorter fiber length and higher pump powers in the simulations. The noisy behavior of the simulated spectra (when compared with the measured spectra) is due to the fact that we report the results of single shot simulations for a given noise seed. We verified that by averaging out several simulations ignited by different noise seeds, the calculated spectra turn out to be even closer to the experiments [19], but this process is time consuming since every simulation takes many hours. We analyzed several simulations with different noise seeds and could notice that the qualitative behavior was the same.

The experiments of Fig. 9 show that the Stokes line was generated at $1407 \mathrm{~nm}$ and the anti-Stokes wave at $855 \mathrm{~nm}$ : these wavelengths match well the analytically predicted values of Eq. (1) (i.e., the Stokes wave at $1416 \mathrm{~nm}$ and anti-Stokes at $852 \mathrm{~nm}$ ). The corresponding FWM phase-matching curves for the four-wave interaction involving the $L P_{01, y}$ and $L P_{11, y}$ pump modes are shown in Fig. 10. The position of the Stokes and anti-Stokes peaks are calculated as a function of the pump wavelength.

When comparing Fig. 9 with Fig. 7, we may notice that in the former case the visible region of the SC spectrum is significantly enhanced by broadband XPM among wavelengths situated on opposite sides of the ZDW (so that they travel at the same group velocity). Such interaction leads to asymmetric spectral broadening, as it was also pointed out in previous works $[2,14,20-22]$.

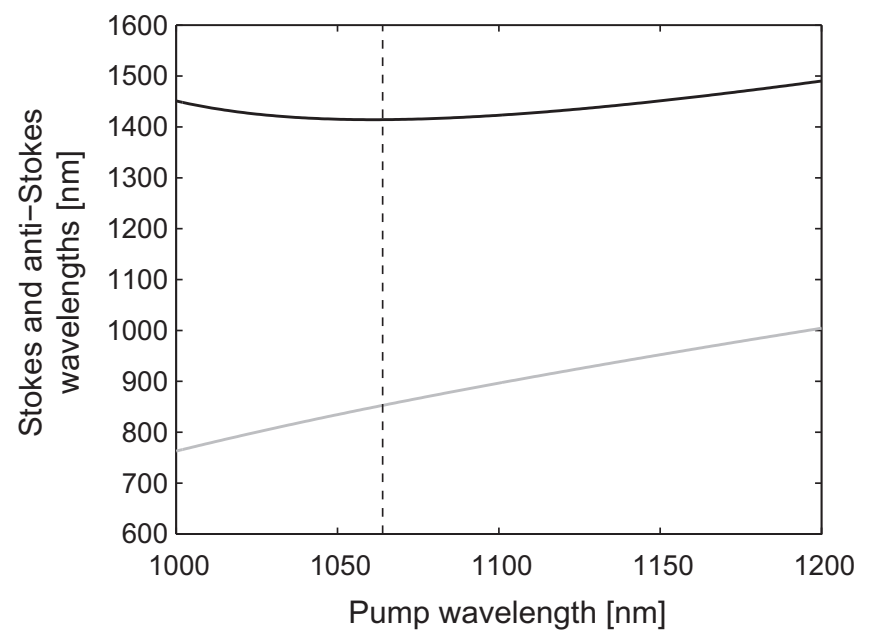

Fig. 10. Position of Stokes and anti-Stokes waves as a function of the wavelength of the pumps $L P_{01, y}$ and $L P_{11, y}$. 
We also studied the impact on SC broadening of varying the input pump power by gradually changing the intensity of the pump beam through an optical attenuator. In Fig. 11 we show the evolution of the spectral broadening as a function of the pump peak power that was coupled in a 2.5 -m-long piece of fiber.

Fig. 11 shows that at relatively low powers the first nonlinear phenomenon that takes place is the MI of the pump, which generates small sidebands closely spaced about the pump wavelength. Whenever the input peak power reaches about $350 \mathrm{~W}$, intermodal FWM occurs and the resulting sidebands start broadening alongside with the pump. Note that the pump and the Stokes sideband mainly broaden towards longer wavelengths owing to Raman scattering. On the other hand Fig. 11 shows that the anti-Stokes FWM peak, because of its vicinity to the ZDW (which is situated at $870 \mathrm{~nm}$ for the $L P_{11, y}$ mode), broadens on its shorter wavelength side as well, eventually reaching the visible spectral region for pump powers above $1 \mathrm{~kW}$. The generation of visible wavelengths
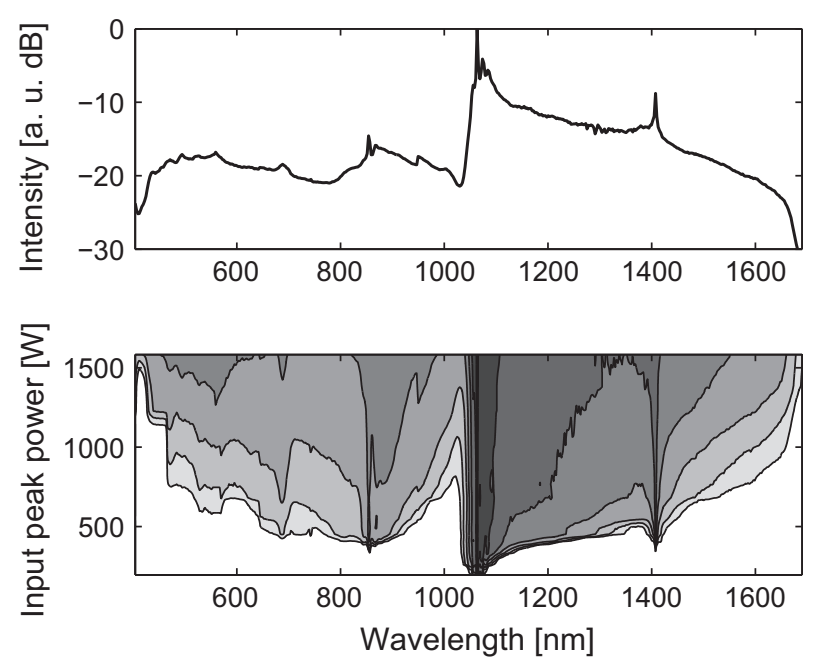

Fig. 11. Experimental output spectra for a 2.5 -m-long fiber versus peak pump power for the $y$ polarization. The peak power was estimated with a broadband power meter at the fiber output. The top figure shows the spectrum at its maximum extent, reaching down to $400 \mathrm{~nm}$ and covering the entire visible region.
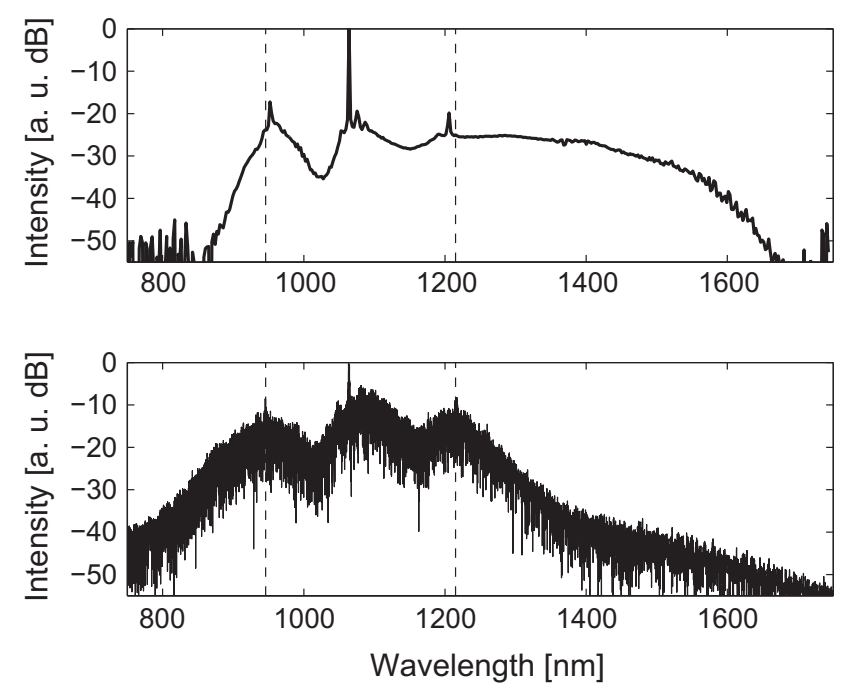

Fig. 12. Experimental (top, fiber length $=2.5 \mathrm{~m}$ ) and numerical (bottom, fiber length $=1.5 \mathrm{~m}, 800 \mathrm{~W}$ per mode) results for the SC spectrum obtained with the joint excitation of the $L P_{01, x}$ and $L P_{11, x}$ modes in the central core; markers indicate the positions of the peaks as predicted by the phase-matching condition. is reinforced by the previously described XPM mechanism [2], and, as represented in Fig. 11 (top), the spectrum reaches the blue at the maximum input power achievable with our source.

Whenever the pump polarization was rotated by $90^{\circ}$ with respect to the $y$ axis, we observed efficient FWM between the $L P_{01, x}$ and $L P_{11, x}$ modes. The maximum peak power coupled inside the MOF was estimated at about $1.7 \mathrm{~kW}$. Quite interestingly, in this case FWM did not lead to the formation of a visible spectrum since the generated sidebands were relatively close to the pump. As shown in Fig. 12, the Stokes and anti-Stokes waves appear at $1206 \mathrm{~nm}$ and $952 \mathrm{~nm}$ respectively: these values are very close to the theoretical values of $1215 \mathrm{~nm}$ and $946 \mathrm{~nm}$ as estimated by the FWM phase-matching condition (Eq. 1).

By varying our launching conditions we could also excite a mixture of all four $L P_{01, x-y}$ and $L P_{11, x-y}$ pump modes. As shown in Fig. 13, this led to the observation of vector modal FWM in addition to the two previously described scalar modal FWM processes along
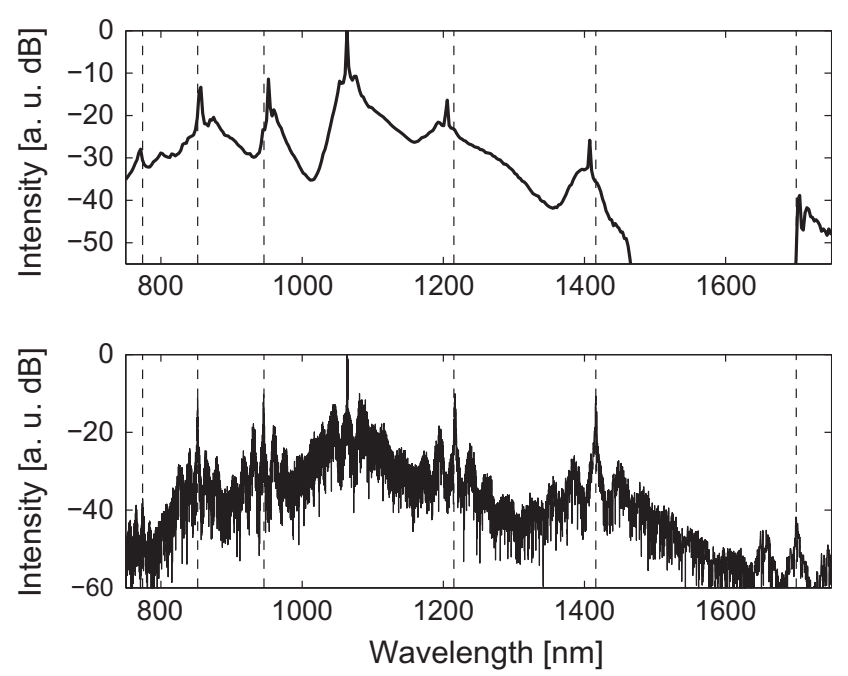

Fig. 13. Experimental (top, fiber length $=1.8 \mathrm{~m}$ ) and numerical (bottom, fiber length $=1 \mathrm{~m}, 400 \mathrm{~W}$ per mode) results for the SC spectrum obtained with the joint excitation of the $L P_{01, x-y}$ and $L P_{11, x-y}$ modes in the central core; markers indicate the positions of the peaks as predicted by the phase-matching conditions.

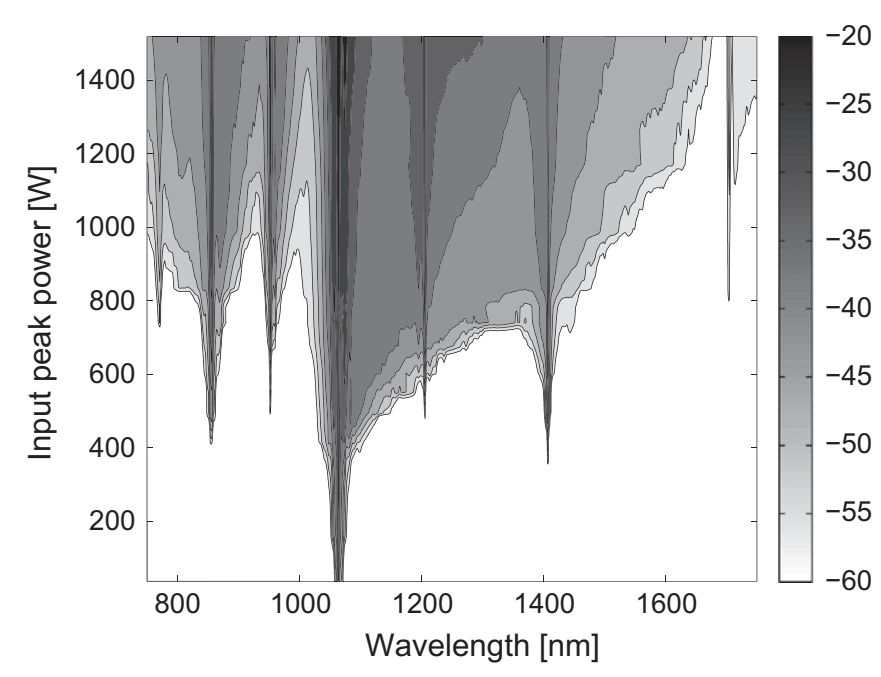

Fig. 14. Experimental output spectra for a 1.8 -m-long fiber versus peak powers for the joint excitation of the $L P_{01, x-y}$ and $L P_{11, x-y}$ modes. The peak power was estimated from the output power measured with a broadband power meter. 


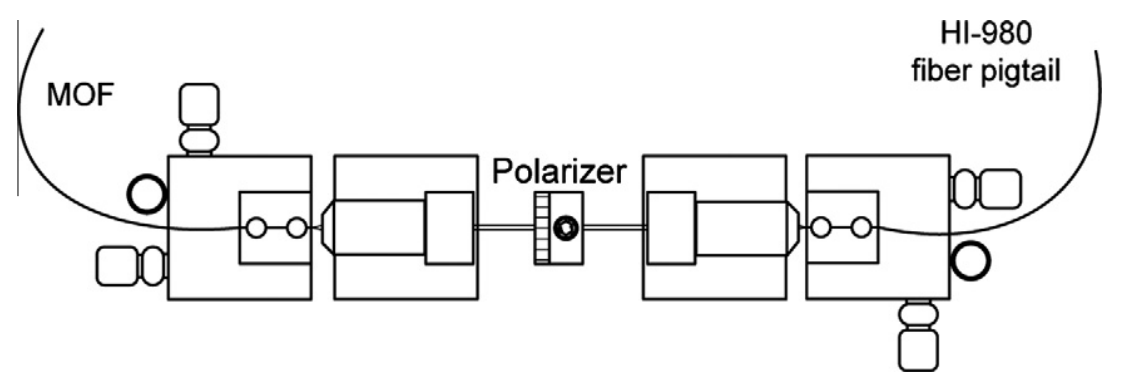

Fig. 15. Scheme of the additional setup used to study the SC polarization. The light coming from the MOF is collimated, passed through a polarizer and it is then refocused into a pigtail of HI980 fiber.

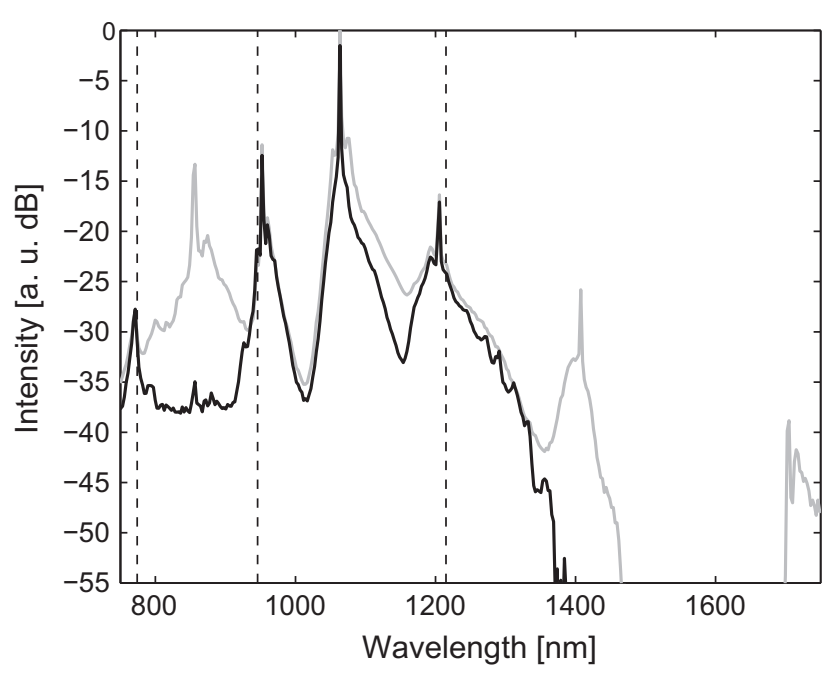

(a) The polarizer passes the $x$ polarization

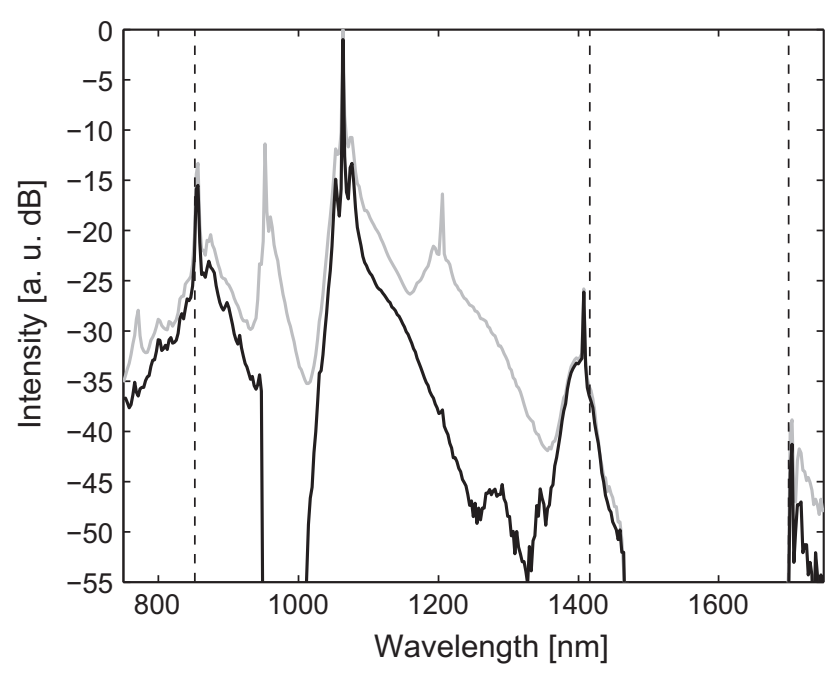

(b) The polarizer passes the $y$ polarization

Fig. 16. (Fiber length $=1.8 \mathrm{~m}$ ) Experimental results for the SC spectrum obtained with the joint excitation of the $L P_{01, x-y}$ and $L P_{11, x-y}$ modes in the central core. In the upper (lower) image, the total spectrum, in gray, is superimposed to the spectrum obtained by passing the $x(y)$ polarization, in black.

the $x$ and $y$ axis. Such vector modal FWM generates two new orthogonally polarized sidebands at the Stokes wavelength $\lambda_{S}=1705 \mathrm{~nm}$ and the anti-Stokes wavelength $\lambda_{A}=771 \mathrm{~nm}$ as described by the following phase-matching equation

$k_{P, 01_{x}}+k_{P, 11_{y}}-k_{S, 01_{y}}(\Omega)-k_{A, 11_{x}}(\Omega)=0$.
The spectrum in Fig. 13, obtained for a fiber length of $1.8 \mathrm{~m}$ with the ANDO AQ-6310B, with a resolution of $2 \mathrm{~nm}$ and 100 averages was compared with the corresponding simulation result from the numerical solution of the four coupled nonlinear Schrödinger equations for each guided mode. As it can be seen, an excellent agreement between experiment and simulation was obtained. By comparing Fig. 13 with Figs. 9 and 12, we can see that the presence of vector modal FWM leads to SC spectrum exhibiting a nearly symmetrical brightness towards its longer and shorter wavelengths. In other words, the presence of two new widely spaced vector sidebands of Eq. (2) leads to a redistribution of pump energy from the NIR into the visible region of the SC spectrum.

In Fig. 14 we show the measured evolution of the SC spectral broadening as a function of pump peak power coupled to a 1.8m-long MOF sample under simultaneous excitation of all four $L P_{01, x-y}$ and $L P_{11, x-y}$ modes.

We finally analyzed the polarization state of the different FWM peaks by collimating the output beam and passing it through a polarizer before focusing into a HI980 fiber to the OSA. A schematic diagram of the additional collimation setup used to study the polarization of the output spectrum is represented in Fig. 15.

The HI980 fiber, which is single mode above $980 \mathrm{~nm}$, was chosen to reduce the effects of modal beating inside the pigtail. We confirmed that the polarization state of the different FWM peaks agrees well with the theoretical prediction of Eq. (2). In Fig. 16 we superimposed the output spectrum measured without the polarizer to the two spectra acquired by selectively passing the $x$ or the $y$ polarization. As can be seen, there is a perfect agreement between theory and experiments: as expected, the peaks at $771 \mathrm{~nm}, 952 \mathrm{~nm}$ and $1206 \mathrm{~nm}$ are polarized along the $x$ axis, while the peaks at $855 \mathrm{~nm}, 1407 \mathrm{~nm}$ and $1705 \mathrm{~nm}$ are polarized along the $y$ axis.

\section{Conclusions}

In this work we performed a complete characterization of the different spectral features that can be obtained with a seven-core MOF. We presented a comprehensive study of the influence of intermodal FWM on SC generation. Our study showed that the most favorable condition for the generation of a broad spectrum that extends from the visible into the infrared is the growth of an anti-Stokes FWM sideband close to the ZDW of the fiber, which broadens towards the short wavelengths because of the interplay among SPM and XPM. We also pointed out that the presence of intermodal vector FWM, involving $L P_{01}$ and $L P_{11}$ pump modes oriented along the fast and slow axes of the central core, respectively, leads to a symmetric redistribution of the input pump energy ranging from the visible to the NIR region of the SC spectrum. The experiments were in excellent agreement with both numerical simulations and with simple analytical estimates based on the phase-matching conditions of the different FWM processes. As a 
potential application of the studied multi-core MOF, we envisage that flexible shaping of a high-brightness SC source could be achieved by the combination of the outputs from its different cores.

\section{References}

[1] J.M. Dudley, G. Genty, S. Coen, Supercontinuum generation in photonic crystal fiber, Rev. Mod. Phys. 78 (2006) 1135-1184

[2] C. Lesvigne, V. Couderc, A. Tonello, P. Leproux, A. Barthélémy, S. Lacroix, F. Druon, P. Blandin, M. Hanna, P. Georges, Visible supercontinuum generation controlled by intermodal four-wave mixing in microstructured fiber, Opt. Lett. 32 (2007) 2173-2175.

[3] A. Labruyère, A. Martin, P. Leproux, V. Couderc, A. Tonello, N. Traynor, Controlling intermodal four-wave mixing from the design of microstructured optical fibers, Opt. Express 16 (2008) 21997-22002.

[4] F.M. Mitschke, L.F. Mollenauer, Discovery of the soliton self-frequency shift, Opt. Lett. 11 (1986) 659-661.

[5] J. Gordon, Theory of the soliton self-frequency shift, Opt. Lett. 11 (1986) 662664.

[6] J. Herrmann, U. Griebner, N. Zhavoronkov, A. Husakou, D. Nickel, J.C. Knight, W.J. Wadsworth, P.St.J. Russell, G. Korn, Experimental evidence for supercontinuum generation by fission of higher-order solitons in photonic fibers, Phys. Rev. Lett. 88 (2002) 173901.

[7] J.M. Dudley, L. Provino, N. Grossard, H. Maillotte, R.S. Windeler, B.J. Eggleton, S. Coen, Supercontinuum generation in air-silica microstructured fibers with nanosecond and femtosecond pulse pumping, J. Opt. Soc. Am. B 19 (2002) 765771.

[8] S. Coen, A. Hing Lun Chau, R. Leonhardt, J.D. Harvey, J.C. Knight, W.J. Wadsworth, P.St.J. Russel, Supercontinuum generation by stimulated Raman scattering and parametric four-wave mixing in photonic crystal fibers, J. Opt. Soc. Am. B 19 (2002) 753-764.

[9] A.V. Gorbach, D.V. Skryabin, Light trapping in gravity-like potentials and expansion of supercontinuum spectra in photonic-crystal fibres, Nature Photon 1 (2007) 653-657.
[10] F. Poletti, P. Horak, Dynamics of femtosecond supercontinuum generation in multimode fibers, Opt. Express 17 (2009) 6134-6147.

[11] M. Lehtonen, G. Genty, H. Ludvigsen, M. Kaivola, Supercontinuum generation in a highly birefringent microstructured fiber, Appl. Phys. Lett. 82 (2003) 2197-2199.

[12] R. Cherif, M. Zghal, L. Tartara, V. Degiorgio, Supercontinuum generation by higher-order mode excitation in a photonic crystal fiber, Opt. Express 16 (2008) 2147-2152.

[13] W.J. Wadsworth, N. Joly, J.C. Knight, T.A. Birks, F. Biancalana, P.St.J. Russell, Supercontinuum and four-wave mixing with Q-switched pulses in endlessly single-mode photonic crystal fibres, Opt. Express 12 (2004) 299-309.

[14] P.A. Champert, V. Couderc, P. Leproux, S. Février, V. Tombelaine, L. Labonté, P. Roy, C. Froehly, P. Nérin, White-light supercontinuum generation in normally dispersive optical fiber using original multi-wavelength pumping system, Opt. Express 12 (2004) 4366-4371.

[15] V. Couderc, P. Leproux, V. Tombelaine, L. Grossard, A. Barthélémy, Raman cascade suppression by using a wide band parametric conversion in large normal dispersion regime, Opt. Express 13 (2005) 8584-8590.

[16] C. Xiong, W.J. Wadsworth, Polarized supercontinuum in birefringent photonic crystal fibre pumped at $1064 \mathrm{~nm}$ and application to tuneable visible/UV generation, Opt. Express 16 (2008) 2438-2445.

[17] G.P. Agrawal, Nonlinear Fiber Optics, third ed., Academic Press, 2001

[18] K.J. Blow, D. Wood, Theoretical description of transient stimulated Raman scattering in optical fibers, IEEE J. Quantum Electron. 25 (1989) 2665-2673.

[19] K. Hammani, C. Finot, B. Kibler, G. Millot, Soliton generation and rogue-wavelike behavior through fourth-order scalar modulation instability, IEEE Photon. J. 1 (2009) 205-212.

[20] V. Tombelaine, A. Labruyere, J. Kobelke, K. Schuster, V. Reichel, P. Leproux, V. Couderc, R. Jamier, H. Bartelt, Nonlinear photonic crystal fiber with a structured multi-component glass core for four-wave mixing and supercontinuum generation, Opt. Express 17 (2009) 15392-15401.

[21] V. Tombelaine, C. Buy-Lesvigne, P. Leproux, V. Couderc, G. Melin, Optical poling in germanium-doped microstructured optical fiber for visible supercontinuum generation, Opt. Lett. 33 (2008) 2011-2013.

[22] J.M. Stone, J.C. Knight, Visibly "white" light generation in uniform photonic crystal fiber using a microchip laser, Opt. Express 16 (2008) 2670-2675. 